\title{
Implementation of Hybrid Algorithm Based on NSCT for Medical Image Fusion
}

\author{
A. S. Srinivasa Rao, M. Bala Krishna, P. Sirish Kumar, Ch. Chandrika, Jaganmohan Rao. T
}

\begin{abstract}
The process of combining the two different modal images into one single image is multimodal image fusion. The resulting image is helpful in the medical field for effective and better detection of disease and the processing of images; surgery, tumor recognition, illnesses, etc. In the only modes of medical images, the merged image attributes cannot be achieved and can be overcome with the image fusion of various modal images.

A new hybrid algorithm for directive multimodal image fusion will be built for this paper based on the non-sub-sampled contourlet transformation. The images will be fuse through the use of the proposed techniques and comparison with existing technological techniques, using quantitative and qualitative measures. MRI and positron-emission tomography (PET) are used. Quantitative steps, like the Entropy (EN) and Structural Similarity Index (SSIM), will be taken to verify the algorithms.
\end{abstract}

Keywords : Entropy Fusion,Image, NSCT, SSIM.

\section{INTRODUCTION}

An image is a 2-dimensional image that typically has a physical object or an individual with a similar appearance to a subject. Image is a 2-dimensional one, like an image, a screen, a three dimensional one, like a statue. Optical instruments, including a lens, mirrors, telescopes, and microscopes, can be captured and natural objects or anomalies like the eye or the surfaces of the body can be captured. More rows and columns, more file sizes and more image resolution. Each and every pixel of an image also increases in terms of size by increasing depth in its color, stores 256 colors in an 8-bit pixel and stores 16 million colors in a 24-bit pixel and the latter in true color.

\section{MEDICAL IMAGE FUSION}

MIF (Medical image fusion) is the registration method and the integration of various images from single or multiple imaging methods to enhance image quality and minimize randomness and duplication, so that medical images may

Revised Manuscript Received on December 13, 2019.

A.S.Srinivasa Rao*, Department of Electronics \& Communications Engineering, Aditya Institute of Technology and Management, Tekkali,Srikakaulam, India. E-mail: asr47@ rediffmail.com.

M.Bala Krishna, Department of Electronics \& Communications Engineering, Aditya Institute of Technology and Management, Tekkali,Srikakaulam,

krishnabala913@gmail.com@gmail.com India.E-mail:

P.Sirish Kumar, Department of Electronics \& Communications Engineering, Aditya Institute of Technology and Management, Tekkali,Srikakaulam, India. E-mail: sirishdg@gmail.com.

Ch.Chandrika, Department of Computer Science \& Engineering, Sarada Institute of Technology and Management, Ampolu ,Srikakaulam, India. E-mail:challachandrika007@gmail.com.

Jaganmohan Rao. T, Department of Electrical \& Electronics Engineering, Aditya Institute of Technology And Management, Tekkali, Srikakaulam, India. E-mail: jaganmohan.tarra@gmail.com. increase their clinical applicability for diagnosis and evaluation. MMIF (Multi-modal image fusion) algorithms and equipment showed remarkable success in improving the clinical reliability of medical picture-based decisions. A comprehensive list of approaches can be found here and the specific scientific issues in the field of clinical image fusion can be summarized.

\section{IMAGE FUSION USING NSCT}

Fusion is necessary for the medical field if an object of modality cannot provide sufficient information itself. For a study, monitoring, diagnosis of diseases and treatment process in one image doctors need high spatial and spectral information. Single modality images cannot obtain this kind of data. In order to solve this, additional information is taken from various modalities of image and fusion is used for integrating such images in different modes like CT, MRI, PET, etc. in order to resolve this issue.

A. Different Fusion Techniques-Comparison: Here we have compared the above methods for medical ima ge fusion shown in table 1 .

B. The Framework for the model:

The framework of the proposed model is shown in figure 1.

C. Transformation:

By examining the figure above, the two medical images captured in two different modes undergo a transformation in the first step. Non-sub-sampled Pyramid NSP is used to decompose a picture to a low frequency and high-frequency portion with 2-channel non-sampled filter banks.The decomposition is subsequently made possible by the low-frequency portion. $\mathrm{K}+1$ subsets will result in one less frequency and one high-frequency image where $\mathrm{k}$ indicates the amount of decomposition. Further high-frequency objects are produced in a non-sub-sampled pyramid (NSP), while the low-frequency image output is only one.

i.A fusion of components with low frequencies: Depending on phase congruency, low-frequency components are combined. Second, the functions are extracted with the phase congruency extractor from the low-frequency sub-images. 
Implementation of Hybrid Algorithm Based on NSCT for Medical Image Fusion

Table 1: Different Fusion techniques comparison

\begin{tabular}{|l|l|l|}
\hline \multicolumn{1}{|c|}{ Fusion method } & \multicolumn{1}{|c|}{ Advantages } & \multicolumn{1}{c|}{ disadvantages } \\
\hline $\begin{array}{l}\text { Principal Component } \\
\text { Analysis (PCA) }\end{array}$ & $\begin{array}{l}\text { With a simple method, the number of dimensions is } r \\
\text { educed with little data loss. }\end{array}$ & Common degradation Produces \\
\hline $\begin{array}{l}\text { Wavelet Transform } \\
\text { (WT) }\end{array}$ & $\begin{array}{l}\text { Reduces spectral distortion and provides better signal } \\
\text { to noise }\end{array}$ & $\begin{array}{l}\text { More spatial resolution of the final fuse } \\
\text { d image. }\end{array}$ \\
\hline Contrast pyramid & There is no possibility of spatial decay & Quality varies according to number \\
\hline
\end{tabular}

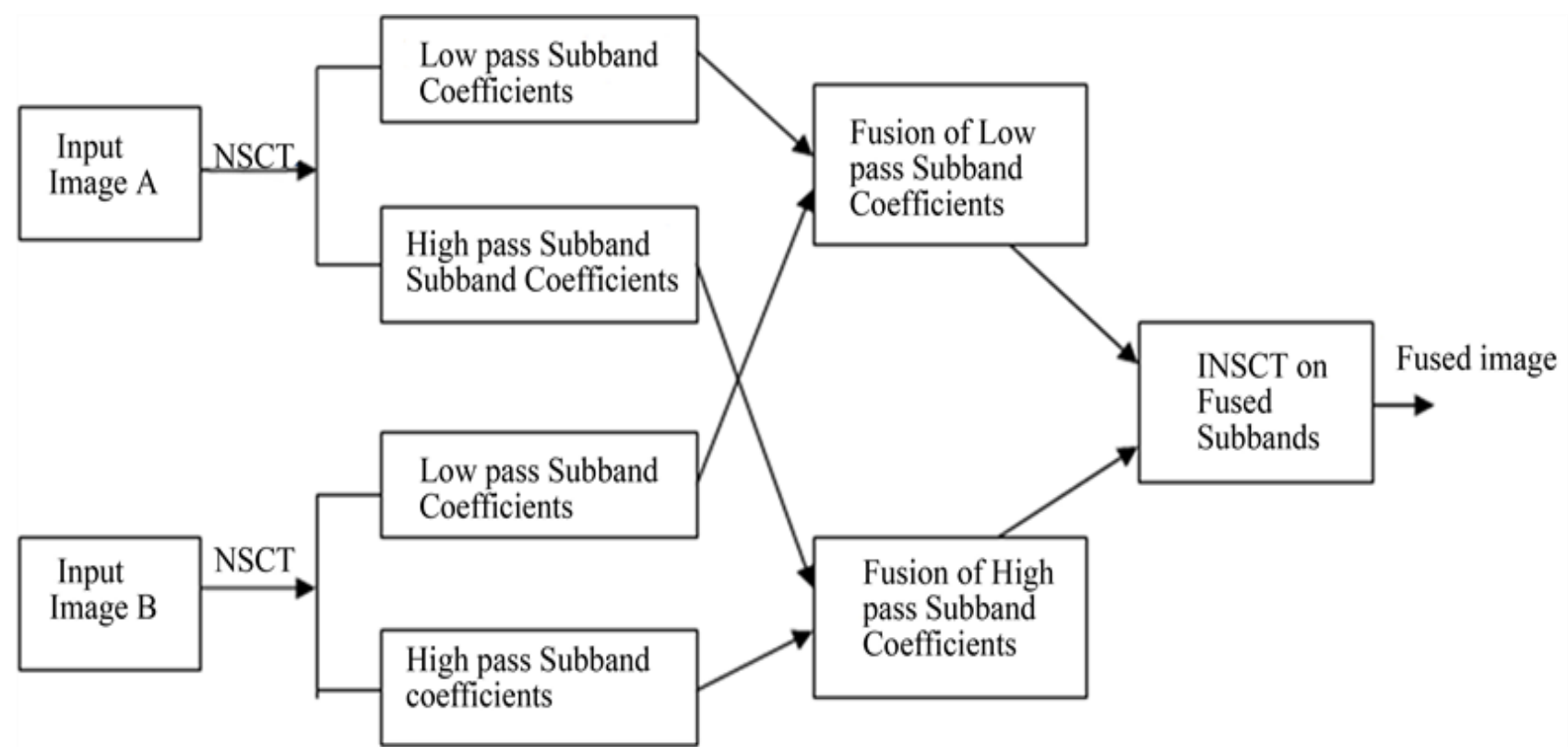

Fig. 1 frame work model for based fusion

ii.A fusion of components with high frequencies: The fusion of components with a high frequency is based on the contrast directive. A notation is denoted for directives for high-frequency sub-images at each scale and orientation and a formulation is determined for direction.

iii.Inverse (I): Perform l-level reverse images to get the image fused in the fused less frequency and high-frequency images.

\section{Enhancement to the Model:}

Multimodal image fusion is of greater importance in the medical field and requires the highest accuracy since it is used for medical examination and disease detection. A three-level fusion is introduced to boost further efficiency. Through a further fusion stage implementation, problems with the domain can be reduced. The principle of high-pass filtering is generalized to wavelet transforms. The DWT application process can be defined as a filter bank. The signal is separated into high and low frequency components at each step of decomposition and the until the desired resolution is achieved, less frequency components can further decompose.

\section{RUN PROCEDURE}

\section{Step Wise Procedure}

Stage 1:

Read the drive for input MRI image.

The image of the storage which you can pick by a single clic $\mathrm{k}$ of a mouse is read in "imread" instruction.

Stage 2:

Displaying the MRI image with the instruction "imshow"On the screen is shown MRI image size $256 * 256$.

Stage 3:

Read the PET image of the storage as second input.

Stage 4:

Shows the PET screen image.

Stage 5:

The filtered MRI image appears on the screen when the Gau ssian LPF is used to input the MRI signal. 
International Journal of Innovative Technology and Exploring Engineering (IJITEE) ISSN: 2278-3075, Volume-9 Issue-2S3, December 2019

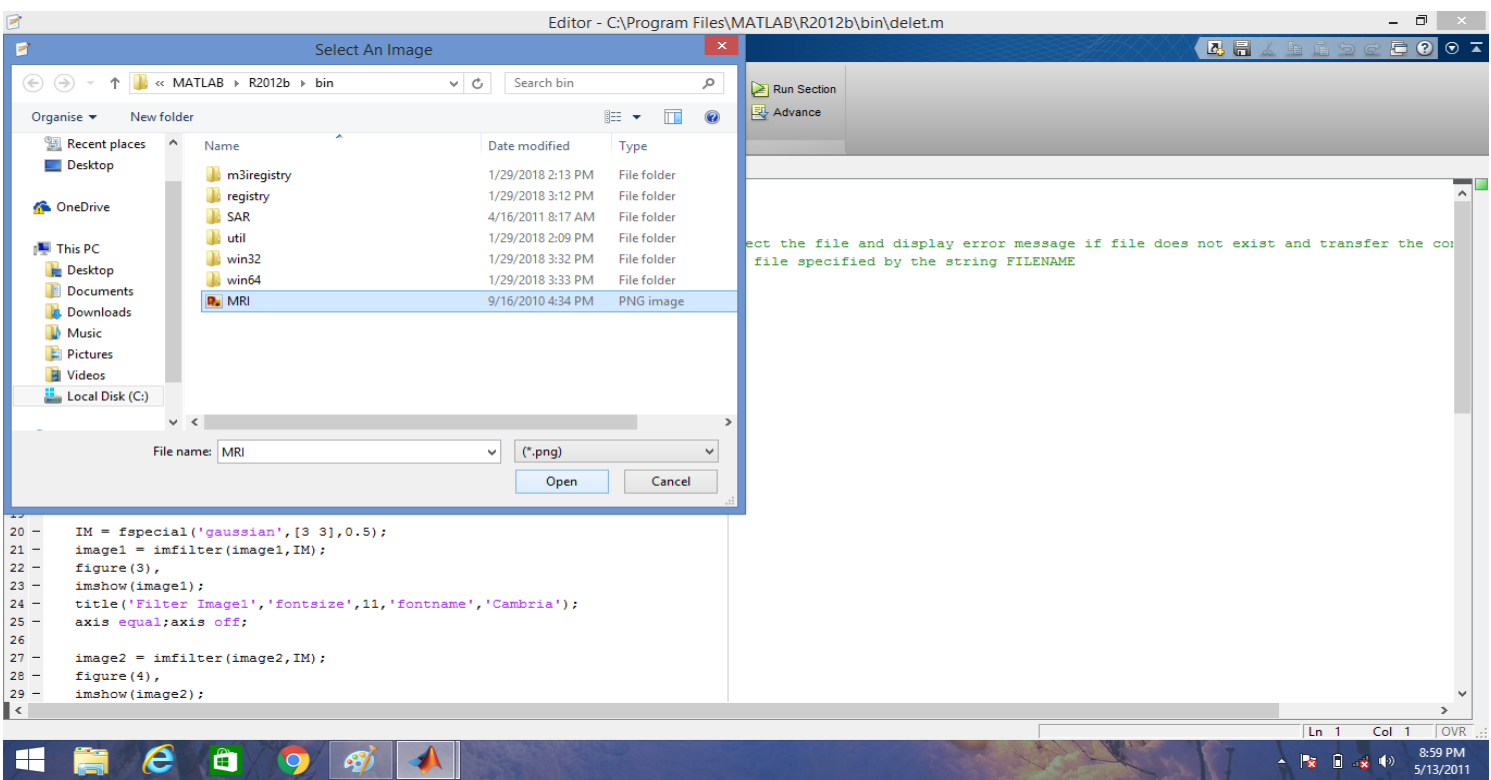

Stage 2:

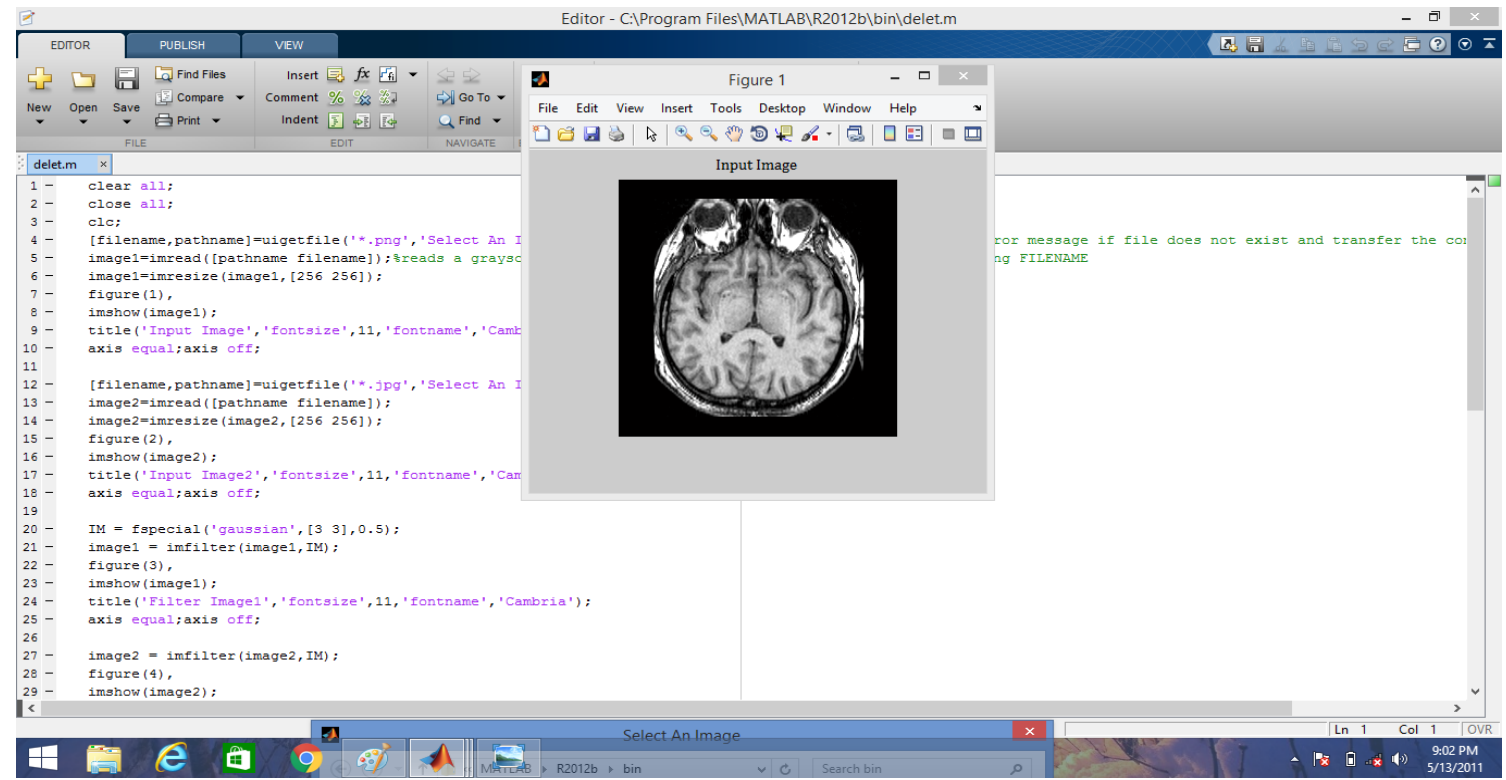

Stage 3:

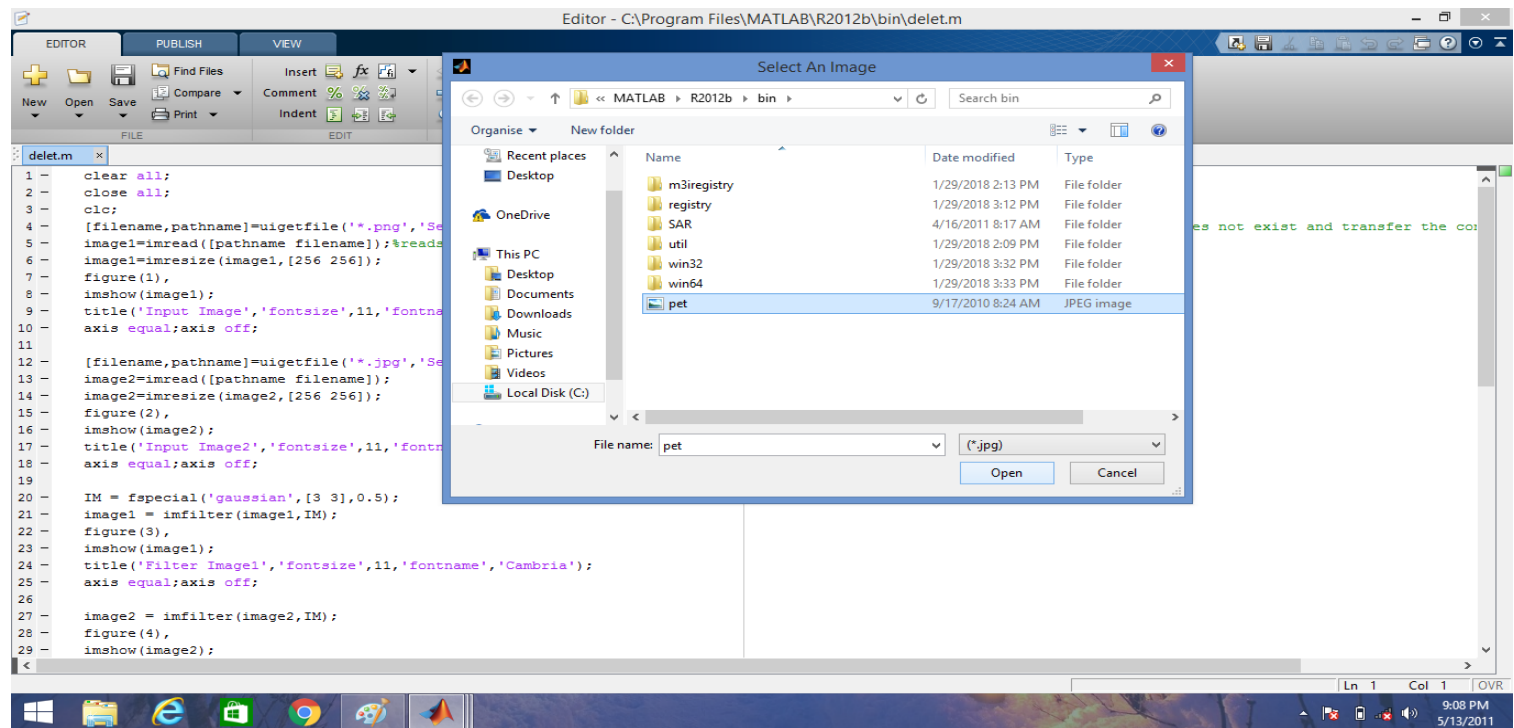

Stage 4: 
Implementation of Hybrid Algorithm Based on NSCT for Medical Image Fusion

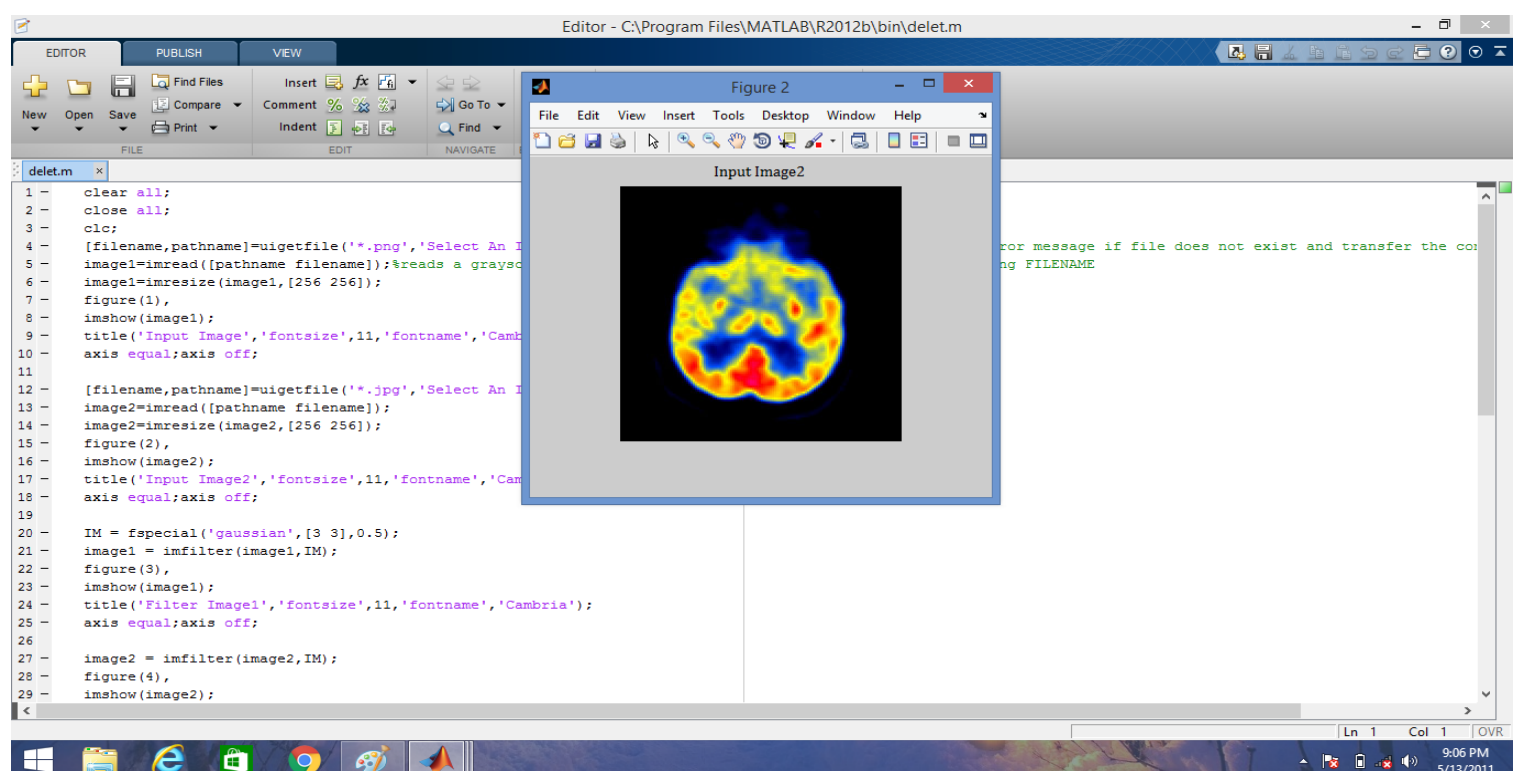

Stage 5:

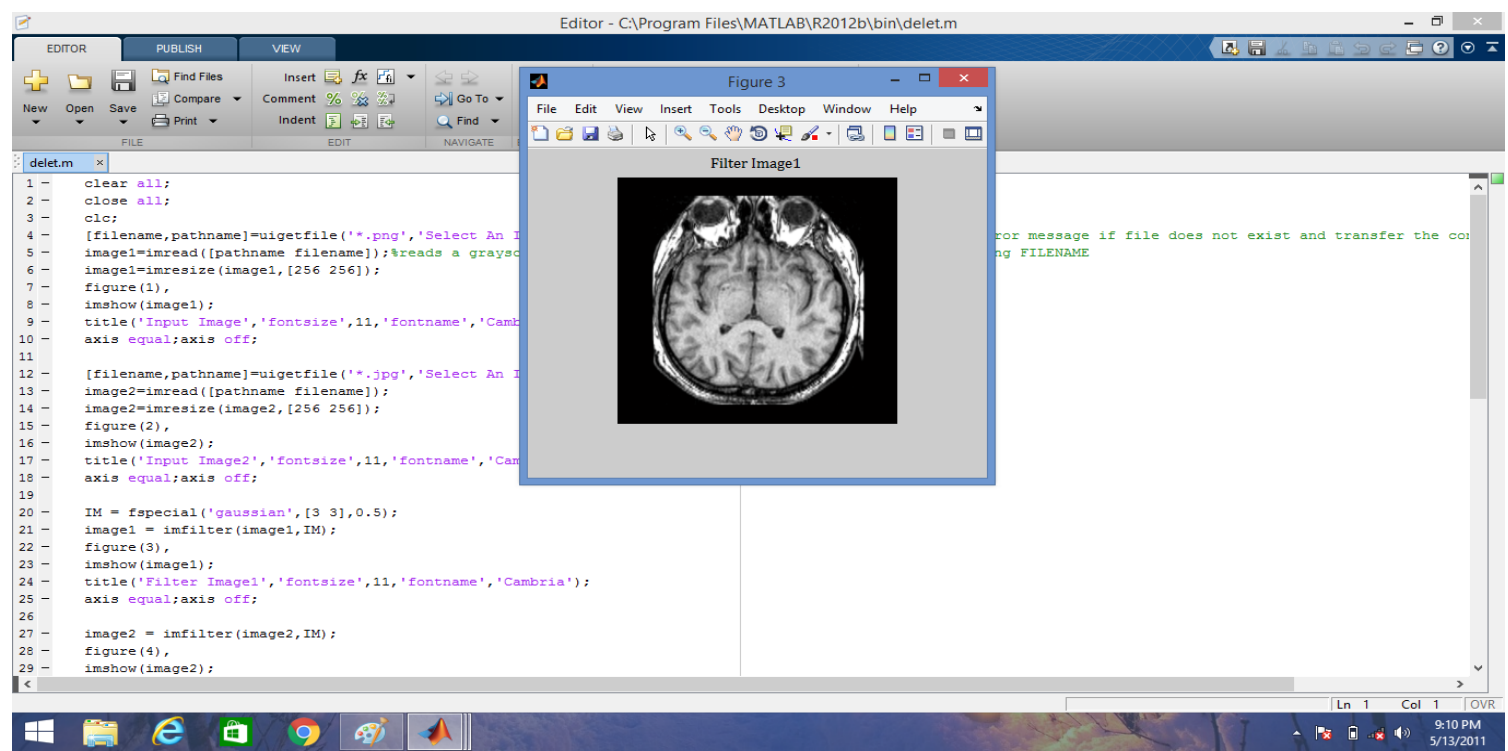

Stage 6:

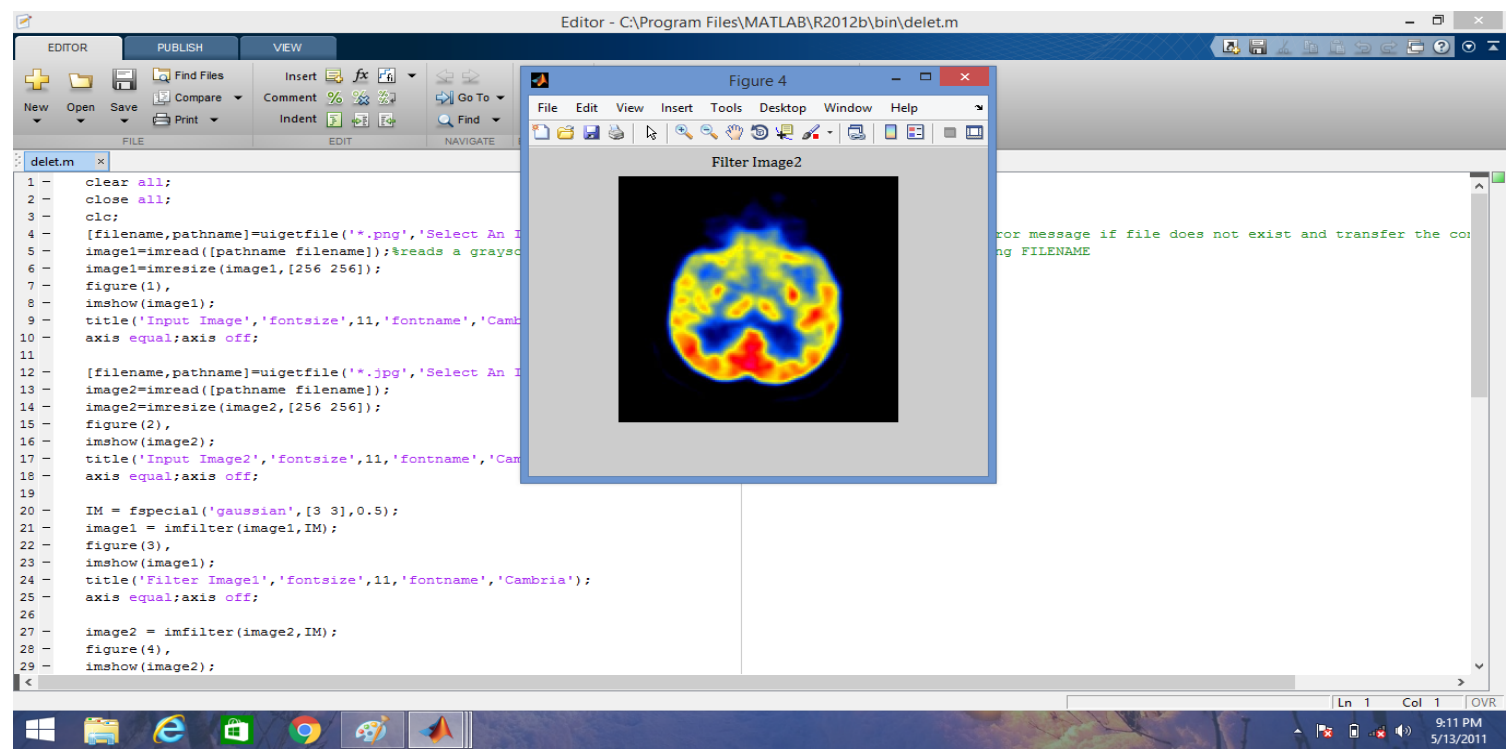

The filtered PET image is shown on the screen, applying Gaussian low-pass filters to the PET image.

Stage 7: 
The decomposition level 1 is shown on the screen Image of non-sub-sampled contourlet coefficients level 1.

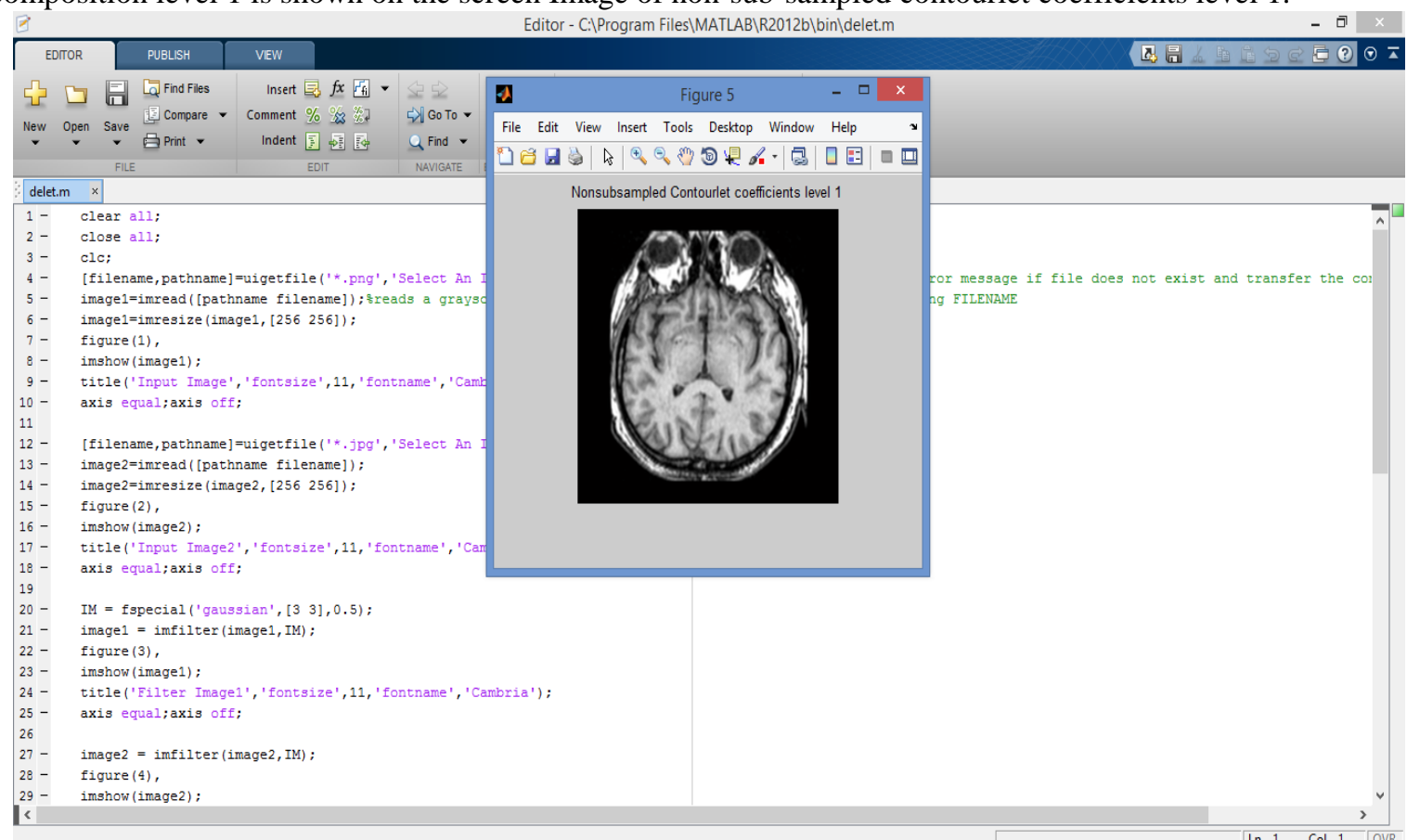

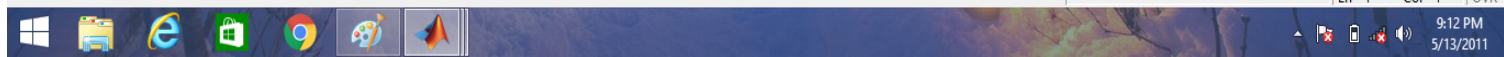

Stage 8:

After this, the weakening of Level 2 is used. The monitor will show the NSCT level 2 coefficients image.

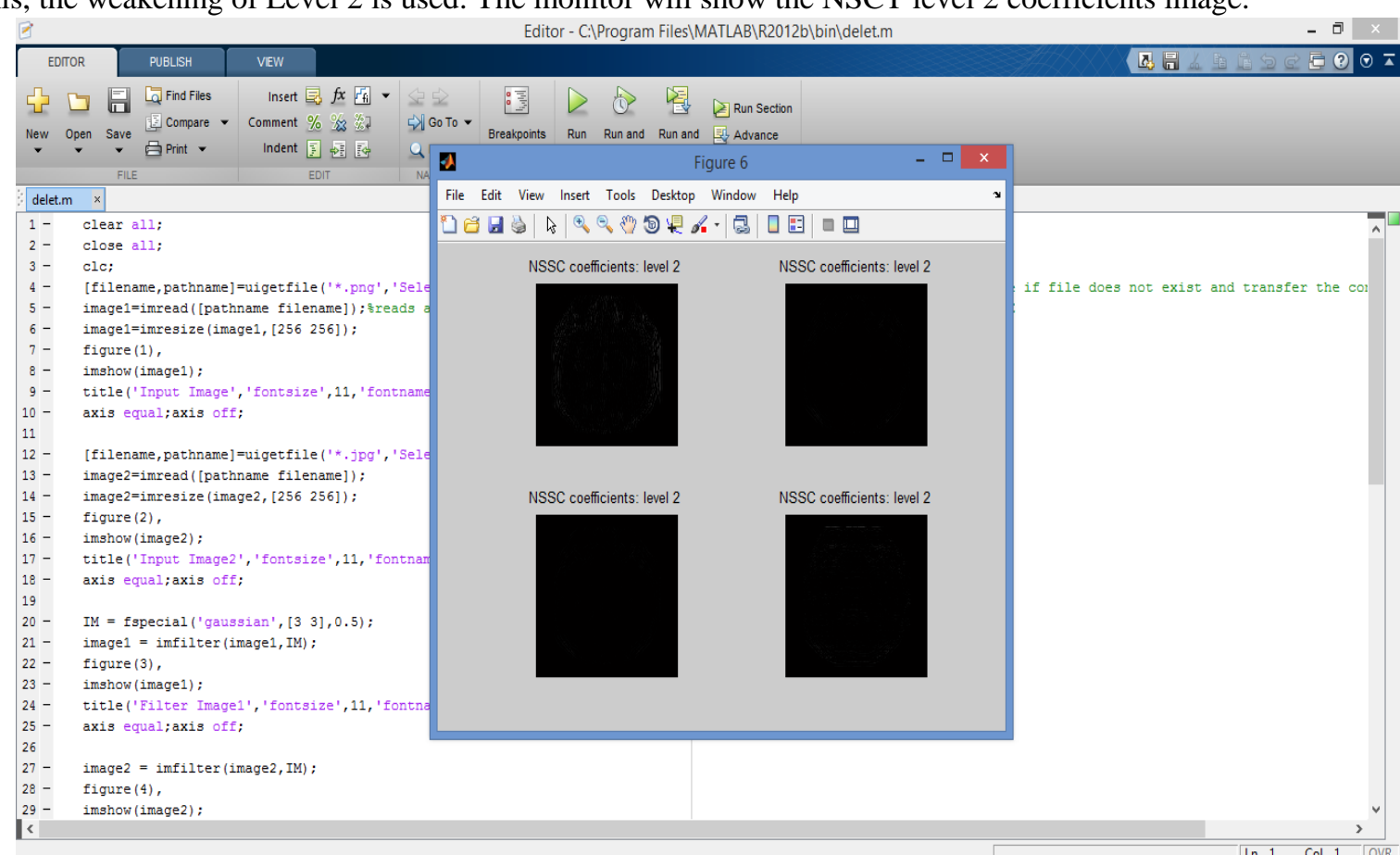

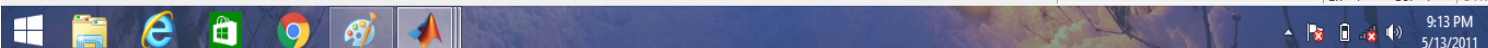

Stage 9:

At this level, approximation fusion is applied. This shows the fused image using 


\section{Implementation of Hybrid Algorithm Based on NSCT for Medical Image Fusion}

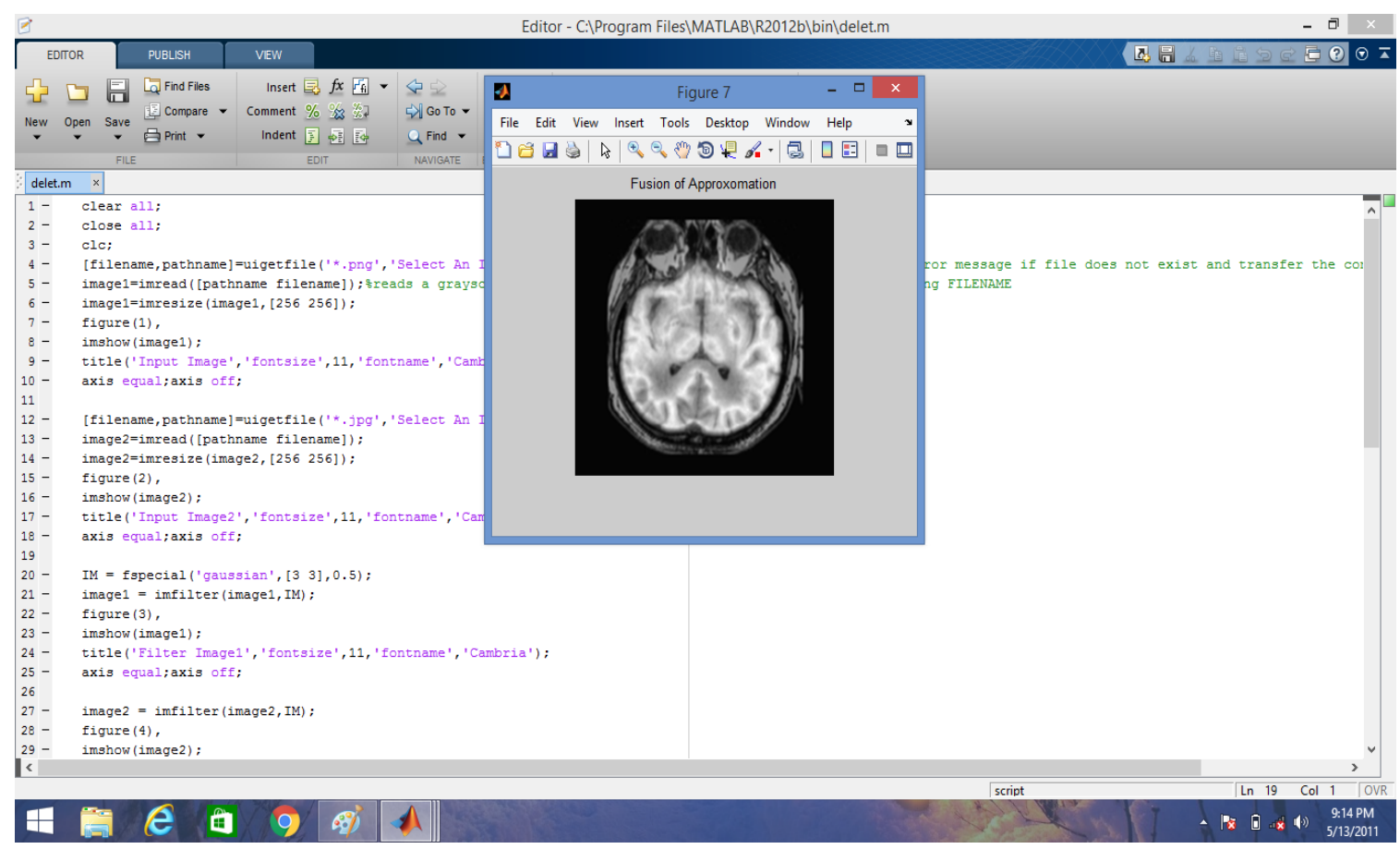

We can fused images in a gray scale since color images consist of red, blue, and green components are difficult to apply. 3 variable parameters are difficult to apply. Thus we apply NSC in gray form that only contains information about intensity (only one parameter).

\section{RESULTS \& DISCUSSIONS:}

Below are the outputs of the NSCT process and wavelet image fusion techniques

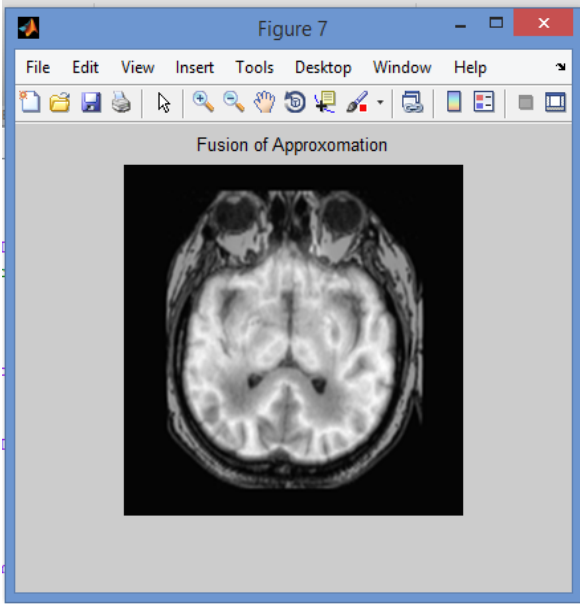

image fusion using non sub-sampled

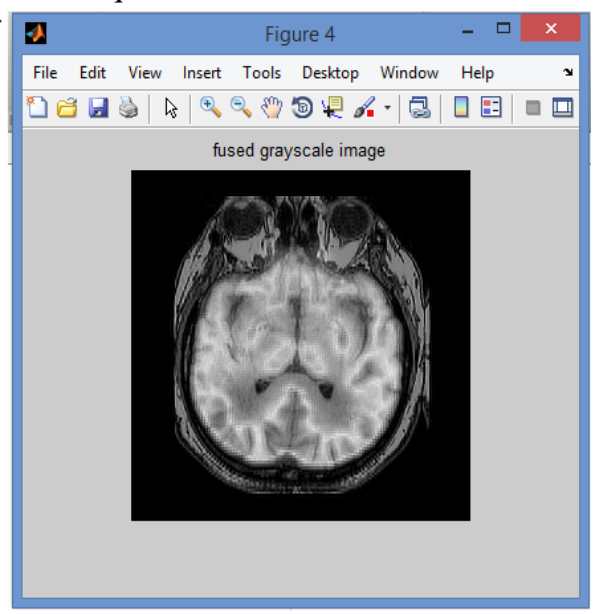

image fusion using discrete wavelet transform

Fig 2: Outputs of the NSCT process and wavelet image fusion techniques

Table 2: Results obtained for two methods

\begin{tabular}{|c|c|c|c|}
\hline Method & Entropy(h) & \multicolumn{2}{|c|}{ SSIM } \\
\hline DWT Fused Image & 3.857 & 07948 & Reference Range: -1 to \\
& 3.93 & 0.0365 & +1 \\
\hline
\end{tabular}

The figure 2 shows clearly the difference between two images through visual evaluation; the output image improved the quality of the picture by reducing blur, increasing the image's contrast. But visual inspection alone is not enough for quality assurance. Those parameters like entropy of both output images and structure-similarity index of two output images with reference MRI-image are therefore calculated internally.
From Table 2, we can note that it allows doctors with clear vision to better identify tissues for diagnosis than dwt-fused image because of high entropy of a fused image. Therefore, for both approaches we have achieved valid values, but more 
SSIM value suggests less deterioration of image quality following image processing. If the structural similarity value with the MRI image is decreased, however, better fusion between MRI and PET can be indicated, and the fused image is better color information than MRI data, resulting in a lower mean SSIM value.

\section{CONCLUSION}

In this paper a novel frame for multi-modal medical images based on the Transform and Directive Contrast Non-Sub-Sampled Contourlet is proposed. For fusion, two distinct rules are used to retain additional information with enhanced performance in the fused image. In our study, the modern fusion algorithms and the proposed systems combine two PET and MR brain images. The graphical and statistical comparisons, such as entropy calculation, Structure Similarity Index (SSIM), show that the algorithm proposed could enhance the object quality by enhancing the visual effect with far less distortion of data.

\section{REFERENCES}

1. B.V.Dasarathy,'Information fusion in the realm of medical applications - a bibliographic glimpse at its growing appea"l, Information Fusion 13(1):1-9, 2012.

2. Gyaourova, A., Kamath, C., \& Fodor,I.K. "Undecimated wavelet transforms for image de-noising". Report, Lawrence Livermore National Lab., CA, pp. 18, 2002

3. Kingsbury, N.G. (2001). "Complex wavelets for shift invariant analysis and filtering of signals". Applied and Computational Harmonic Analysis, Vol. 10, No. 3, pp. 234-253.

4. I.W. Selesnick, "The Double-Density Dual-Tree DWT," IEEE Trans. Signal Processing, vol. 52, no. 5, pp. 1304-1314, May 2004.

5. Gonzalez, R. C. and Woods, R. E. and Eddins, S. L.”Digital Image Processing Using MATLAB”, Prentice Hall, 2004.

6. S. G. Mallat, "A Theory for Multiresolution Signal Decomposition: The Wavelet Representation", IEEE Trans. on Pattern Analysis and Machine Intelligence, 11(7), 1989, 674-693.

\section{AUTHORS PROFILE}

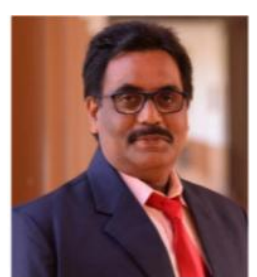

Dr. A. S. Srinivasa Rao is currently working as a Professor \& Principal with Dept of ECE, Aditya Institute of Technology and Management, Tekkali,Srikakulam. He completed his B.E and M.Tech from AU College of Engineering; Visakhapatnam . He also completed his Ph.D from Andhra University with Antennas as research area. He is a Life member of IETE, IETE, IE and member of IEEE. He has more than 40 publications in various national, international conferences and Journals.

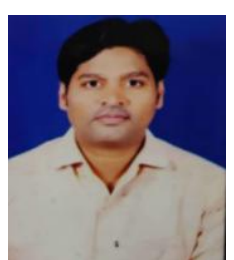

M.Bala Krishna received his M.Tech degree in VLS Design from ANNA University, Chennai. He is currently working as a Sr.Assistant Professor in Aditya Institute of Technology and Managemen, Tekkali, Srikakulam. He is currently doing Ph. D. from AU College of Engineering, Visakhapatnam. His research interests are Signal Processing and embedded systems. He has 10 publications in various national, international Journals.

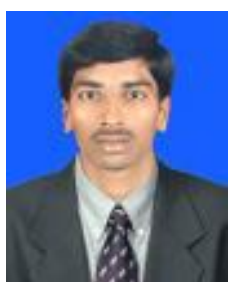

P.Sirish Kumar received M.Tech degree in VLS System Design from Aditya Institute of Technology \& Management,Tekkali, Srikakulam. He is currently working as an Assistant Professor in Aditya Institute of Technology \& Management,Tekkali, Srikakulam. He is currently doing Ph. D. from GITAM University, Visakhapatnam. His research interests are global positioning system and embedded systems. He has 20 publications in various national, international Journals.

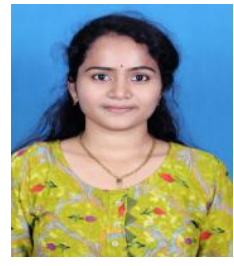

Ch.Chandrika received her B.Tech from IIIT, Nuzvid and M.Tech degree in Computer Science Engineering from Aditya Institute of Technology \& Management,Tekkali, Srikakulam. She is currently working as an Assistant Professor in Sarada Institute of Technology \& Management, Ampolu, Srikakulam.

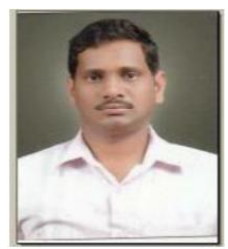

T.Jaganmohan Rao received M.Tech from AU College of Engineering, Visakhapatnam. He is currently working as an Assistant Professor in Aditya Institute of Technology \& Management,Tekkali, Srikakulam.. He has 15 publications in various national, international Journals. 